\title{
Un nuevo cálato inscrito de Ca n’Oliver (Cerdanyola del Vallès, BARCELONA)
}

A New Inscribed Kalathos from Ca n'Oliver (Cerdanyola del Vallès, Barcelona)

NOEMÍ MONCUNILL MARTÍ1 (D), JOAN FRANCÈS²

(1) Université Paris Sorbonne. nmoncunill@gmail.com

(2) Museo y poblado ibérico de Ca n'Oliver. FrancesFJ@cerdanyola.cat

Resumen:

Este artículo presenta la edición de una nueva inscripción ibérica hallada en el yacimiento de Turó de Ca n'Oliver (Cerdanyola del Vallès, Barcelona). El nuevo texto documenta una nueva variante de la fórmula de la autoría en ibérico, que se caracteriza por la aparición de la palabra ekiar/egiar. En este caso, sin embargo, dicha fórmula presenta algunas variantes morfológicas y fonéticas dignas de comentario.

Palabras clave: epigrafía paleohispánica, Hispania, lengua ibérica, onomástica, Ca n'Oliver.

\section{Abstract:}

This paper presents the edition of a new Iberian inscription found in Turó de Ca n'Oliver (Cerdanyola del Vallès, Barcelona). This inscription can be interpreted as a variant of the well-known authorship formula in Iberian, which is characterised by the presence of the word ekiarl egiar. However, in this particular case this formula shows some interesting morphological as well as phonetical features which make it unique.

Key words: Palaeohispanic epigraphy, Hispania, Iberian language, onomastics, Ca n'Oliver. 


\section{INTRODUCCIÓN ${ }^{1}$}

La expresión de la autoría en ibérico despertó ya tempranamente el interés de los especialistas, principalmente por el llamativo parecido de su núcleo, la palabra egiar $^{2}$, con el verbo vasco egin 'hacer' (Beltrán 1942: 51; Gómez Moreno 1945: 281). Dejando a un lado la controvertida comparación del ibérico con el vasco ${ }^{3}$, el hecho es que también el estudio tipológico de las inscripciones, así como el análisis combinatorio y distribucional de los textos han ido confirmando la validez de esta interpretación: la fórmula de autoría parece expresarse en ibérico con un nombre personal, ocasionalmente sufijado con -de, seguido de la mencionada palabra egiar (MLH III.1 § 570; de Hoz 2001: 354; Rodríguez Ramos 2004: 274-276; Quintanilla 2005: 510; Ferrer 2006: 154-156; Moncunill 2007: 156-158; de Hoz 2011: 296-313). Esta estructura se documenta por el momento en más de veinte inscripciones, la mayoría de ellas realizadas en el momento de fabricación de la pieza y en objetos de producción local.

En este trabajo presentamos una nueva inscripción inédita procedente del oppidum Turó de Ca n'Oliver (Cerdanyola del Vallès, Barcelona) que documenta una variante de la descrita fórmula de autoría. El texto presenta por sí mismo un interés notable, al reunir ciertas particularidades tanto gráficas como morfológicas y fonéticas dignas de comentario. Además, este nuevo testimonio resulta también destacable por el hecho de que, con ésta, ascienden ya a tres las variantes de esta fórmula documentadas en el mismo yacimiento.

\section{EL POBLADO IBÉRICO DEL TURÓ DE CA N'OLIVER, CERDANYOLA DEL VALLÈS}

El yacimiento ibérico del Turó de Ca n'Oliver se encuentra en el municipio de Cerdanyola del Vallès, en una de las primeras elevaciones de la vertiente vallesana de la sierra de Collserola. Las intervenciones de los últimos años han permitido profundizar en el conocimiento de la evolución urbanística y en la secuencia cronológica de la ocupación. Se trata de un poblado que vertebra un amplio territorio con una notable potencialidad agrícola y ganadera, ejerciendo como centro de captación y redistribución de la producción proporcionada por los pequeños asentamientos dispersos por el llano. La aparición de campos de silos sería un claro reflejo de este fenómeno de acumulación (Francès y Guàrdia 2011: 267-291).
En Ca n'Oliver la singularidad de algunos materiales, como las cráteras de columnas, la abundancia de armamento y el utillaje de hierro, junto con la presencia de elementos heroizantes o apotropaicos, como los fragmentos escultóricos identificados como leones (Barrial y Francès 1985) y una estela del denominado Grupo del Bajo Aragón (Margenat 2015), indicarían la presencia de personajes de cierta posición social.

La evolución del asentamiento ibérico se puede sintetizar en cuatro grandes fases. La primera ocupación del cerro (fase 0 ) estaría representada por una fase inicial previa a la propiamente urbana. Estaría formada por una agrupación de cabañas semiexcavadas en la roca de la cuales sólo se conservan algunos recortes y agujeros de poste. La ausencia de materiales asociados claramente diferenciables de los más antiguos de la fase 1 determina que la datación sea muy problemática. A pesar de ello, se puede situar en un momento anterior al último cuarto del s. VI a.C.

La primera fase propiamente urbana del asentamiento (fase 1) habría que datarla entre el último cuarto del s. VI y mediados del s. V a.C. ya en un contexto cultural inequívocamente ibérico. En esta fase se construyen los primeros barrios con las técnicas constructivas propias de este horizonte, con casas de planta rectangular, de dimensiones modestas y de una gran simplicidad, aparecen compartimentadas, con muros formados por un zócalo de piedra y el alzado de adobes. Es interesante destacar el hecho de que, desde el inicio, el asentamiento ocupaba una notable superficie.

La segunda fase comienza a mediados del s. V a.C. El poblado es objeto de una profunda reforma constructiva y experimenta un cambio radical en la concepción del espacio de habitación. En este momento las antiguas habitaciones de la fase 1 son amortizadas y sobre ellas se construyen nuevas casas, más amplias, que se apoyan en un potente muro de cierre que delimita asentamiento.

Durante la fase 3 , datable entre finales del s. IV y finales del s. III a.C., se producen nuevas reformas. En esta fase se refortifica el poblado con la construcción de nuevos accesos y, posiblemente, de un conjunto de defensas exteriores formadas por diversas torres y tramos de foso. Habrá que relacionar estas reformas con la consolidación de Ca n'Oliver como centro de acumulación de la producción, como indica la presencia de campos de silos situados tanto en el interior como en el inmediato exterior del poblado. Respecto a la cronología de estos depósitos, el más antiguo se puede datar a finales del s. IV o inicios del III a.C., es decir, en un momento inicial de la fase. 


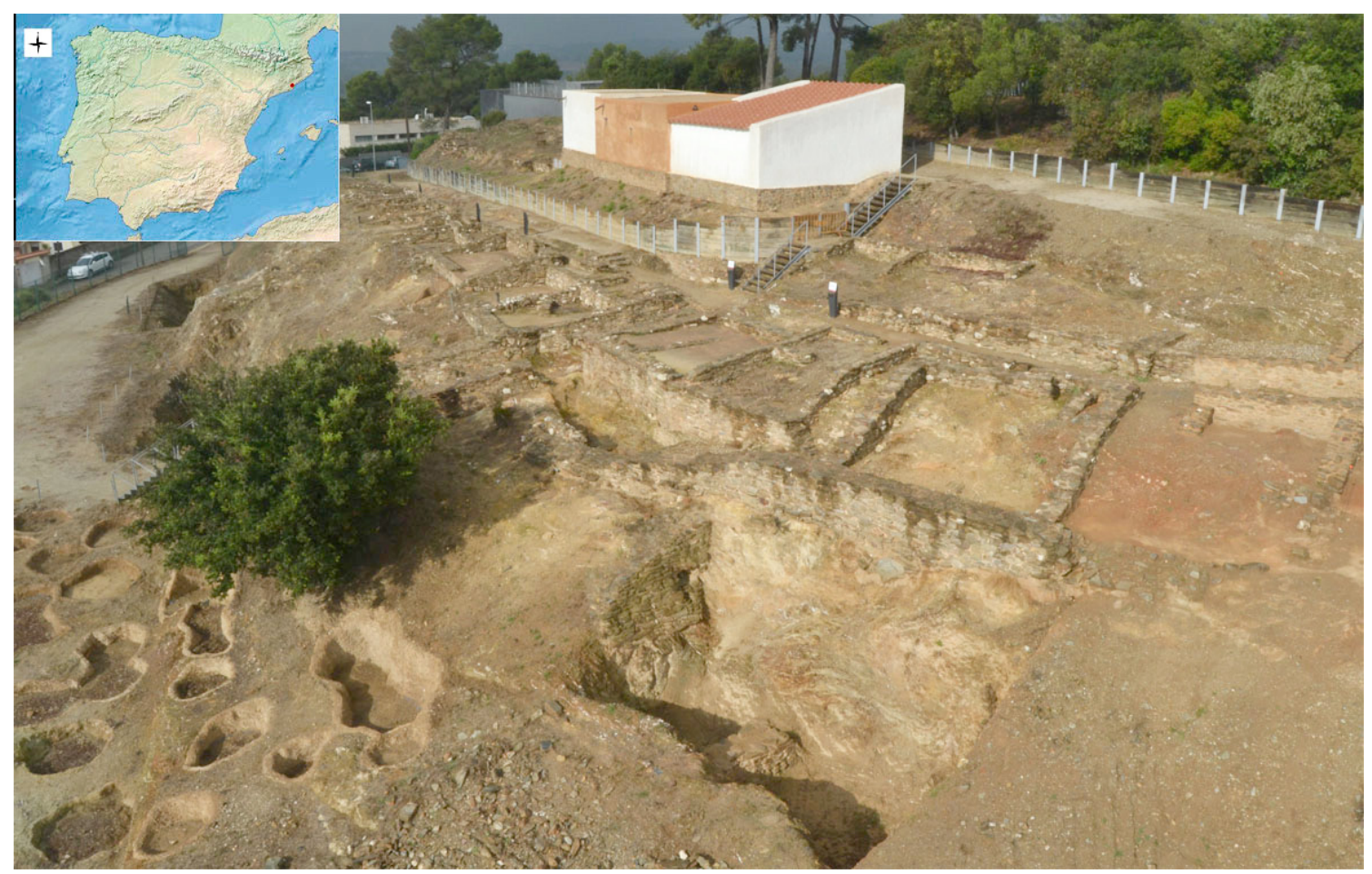

Fig. 1: Imagen general de las excavaciones de Turó de Ca n’Oliver en la que se muestra el foso FS-635, donde apareció la inscripción. Fotografía tomada desde el S.

Ca n'Oliver se consolidará durante el periodo del Ibérico Pleno (fases 2 i 3) como centro artesanal y de intercambio de mercancías. Existen claras evidencias de espacios destinados al trabajo metalúrgico y, en las últimas intervenciones, también se han localizado en el interior de los fosos defensivos, abundantes escorias cerámicas y piezas aparentemente pasadas de cocción. Se trata de evidencias indirectas de la existencia de, como mínimo, un horno de cerámica cercano del que tenemos referencias orales y fotografías antiguas, aunque todavía no se ha podido localizar. El final de este momento de expansión del poblado se produce de manera abrupta ya que Ca n'Oliver es destruido en el marco de los acontecimientos bélicos relacionados con la Segunda Guerra Púnica.

Una última fase, ya dentro de un contexto de romanización (fase 4) y datada a partir de los primeros decenios del s. II a.C., comportará una nueva reorganización urbana del asentamiento. Destaca la construcción de un nuevo muro defensivo, la ampliación y continuidad del campo de silos exterior y la construcción de nuevas casas tanto en el interior del poblado como extramuros. A finales del s. II a.C. los síntomas de decadencia empiezan a ser evidentes y hacia el 50 a.C. el yacimiento es abandonado definitivamente poniendo fin a la ocupación que había durado 500 años casi sin interrupción.

\section{DESCRIPCIÓN DE LA PIEZA Y LECTURA DE LA INSCRIPCIÓN}

La pieza que presentamos fue hallada durante la campaña de excavaciones realizadas en el poblado del Turó de Ca n'Oliver ${ }^{4}$ en el año 2013, en los niveles de amortización de los fosos defensivos del poblado (foso FS-635), datables en el s. III a.C. o principios del II a.C. Se trata de un fragmento de cálato de cerámica ibérica común de cocción oxidante, cuyas medidas máximas son 17,5 x 8,5 cm; la inscripción fue realizada ante coctionem en el labio y la medida de los signos es de $c a .1 \mathrm{~cm}$.

El texto está escrito en signario ibérico nororiental y corre, como es habitual en este sistema de escritura, de izquierda a derecha. Aunque no se observan duali- 


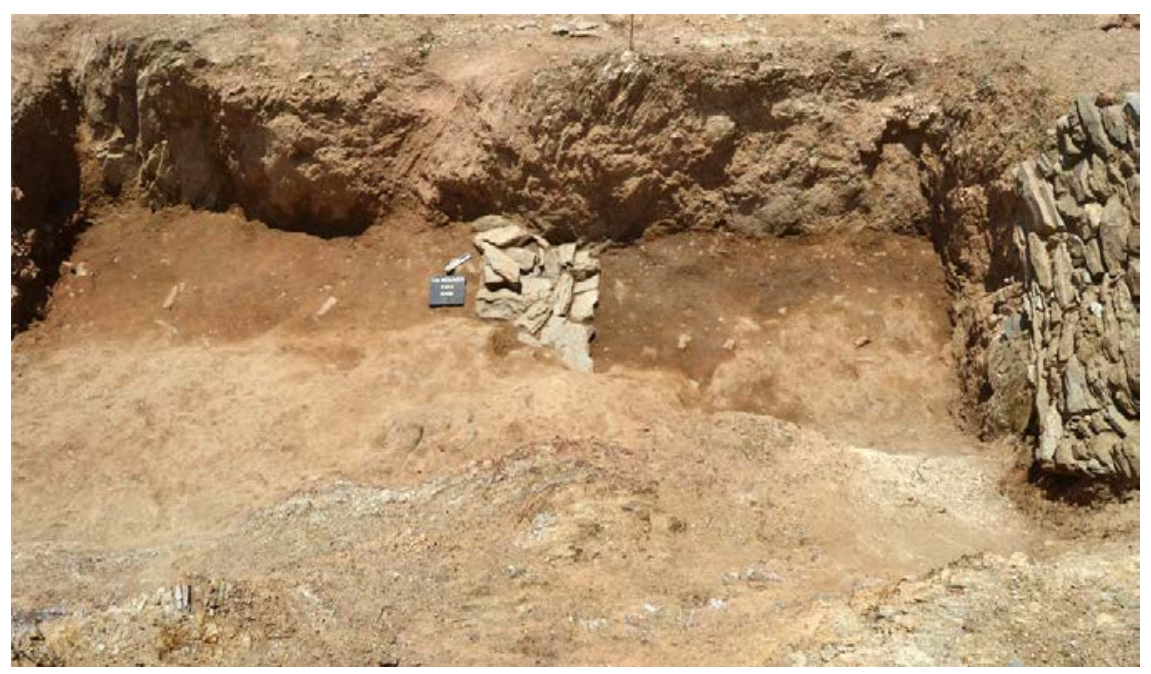

Fig. 2: Detalle del foso FS635 , donde apareció la inscripción. Fotografía tomada desde el N.

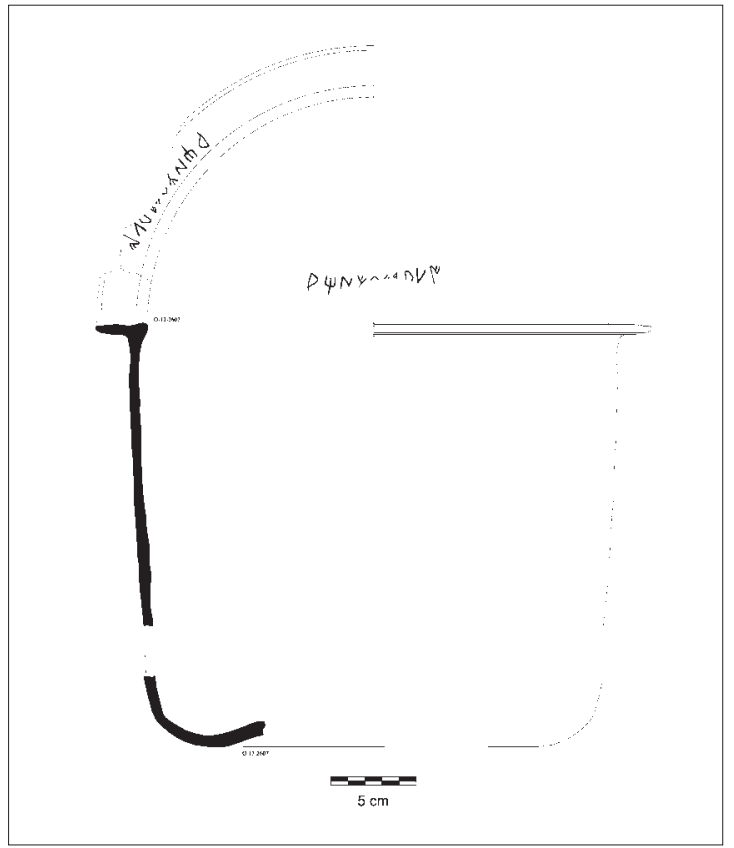

Fig. 3: Perfil del cálato con inscripción.

dades explícitas en la serie de las oclusivas, la presencia de los signos ta y ki complejos es indicio suficiente para considerar que la variante usada es la denominada "dual". Este hecho invita a una datación a finales del s. III o principios del II a.C. (Ferrer 2005: 957-982), cronología que coincide con los datos contextuales del hallazgo.
A pesar de la fragmentariedad de algunos de los signos, no se plantean dudas de lectura importantes, salvo quizás para el signo 5 , cuya transcripción más probable es ŕ, sin que pueda, sin embargo, excluirse drásticamente una lectura como te o ku, signos que presentarían, sin embargo, un módulo inferior al del resto y que resultan, por consiguiente, menos factibles; como veremos a continuación, también los paralelos onomásticos aconsejan leer ŕr. En consecuencia, es posible reconocer los alógrafos siguientes, según la clasificación propuesta por J. Untermann en $M L H$ III.1: a6; ti1; n1; bo 3 = ta (Ferrer 2005); ́r 3; e e 4 ?; ki9; a6; ḿ $4 ; \mathbf{i} 1$. La lectura que proponemos es:

\section{adintaŕekiaḿi}

\section{ANÁLISIS LEXICAL E INTERPRETACIÓN DEL TEXTO}

La interpretación del contenido del texto se ve facilitada por la existencia de numerosos paralelos en el léxico ibérico. A pesar de la inexistencia de interpunciones, es así posible identificar tres unidades léxicas, que comentaremos a continuación: adintaŕ, ekia y mí.

adintaŕ: es un antropónimo ibérico regular, formado con los elementos adin (MLH III.1, §7.19) y taŕ (MLH III.1, §7.115). El único hecho destacable es que no presenta sufijación alguna, como sí sucede con la mayoría de las inscripciones de autoría, en las que el nombre personal va seguido del morfo -te/-de, marcando plausiblemente el agente de la acción (Velaza 2002; Luján 2010: 
Fig. 4: Fotografía de la inscripción realizada en el labio del cálato.

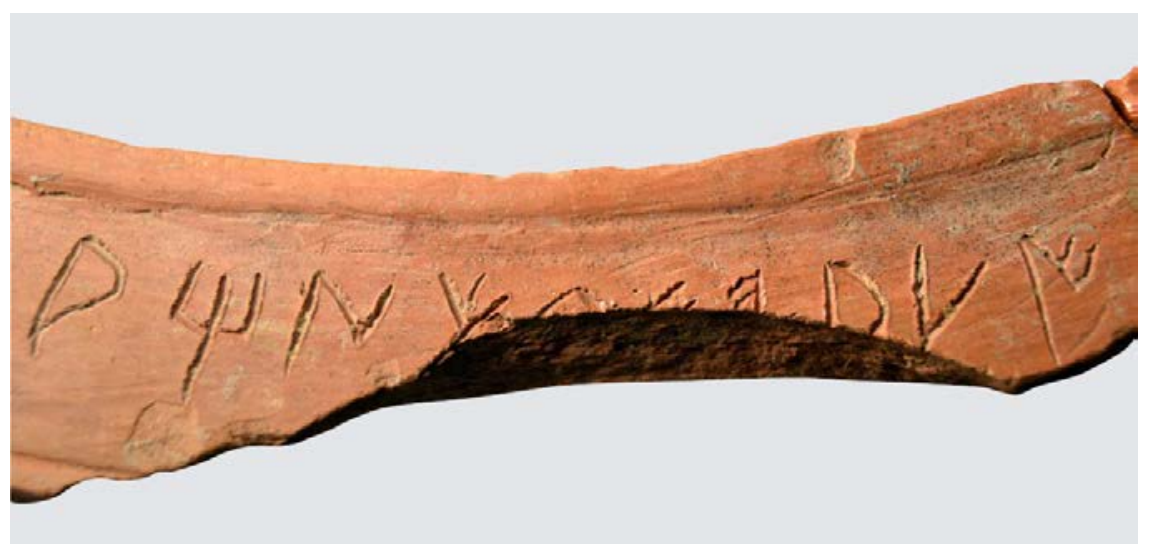

Fig. 5: Dibujo de la inscripción.

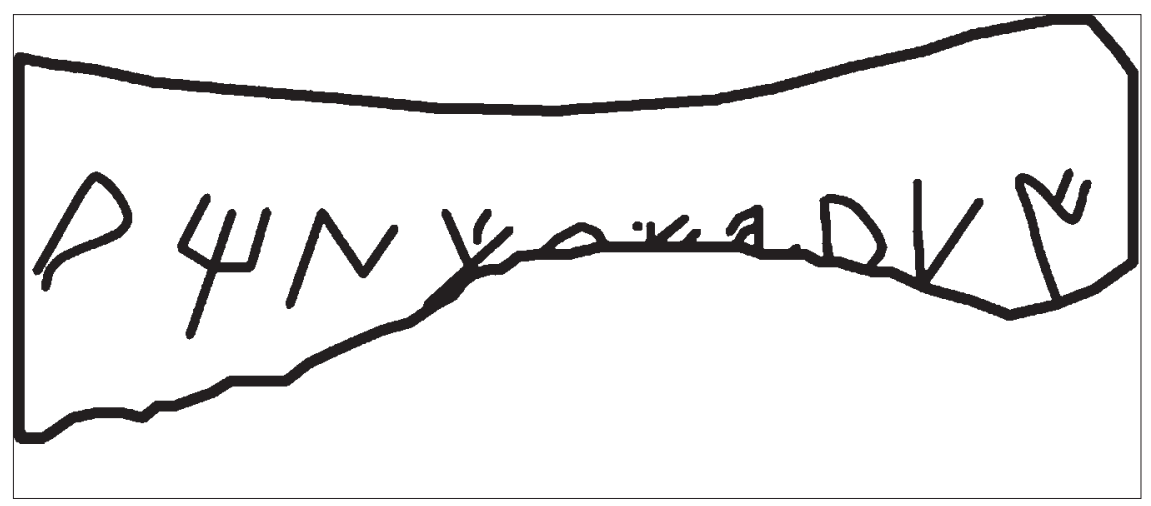

291-298). Así es, por ejemplo, en el conocido mosaico de La Caridad (Caminreal) (E.7.1): likinete ekiar usekeŕteku. Existen, sin embargo, al menos tres otros ejemplos en los que esta misma fórmula podría ir encabezada por un nombre sin sufijo ${ }^{5}$ : ban [sel]kiskeŕ ekiar... (F.13.6), según lectura de Moncunill 2017; bal]tuśerban alâku ekiar... (F.13.7), según propuesta de lectura de Ferrer y Escrivà (2013: 466) e iŕe keśtaḿn ataŕśu ekiar sinekun (Panosa 1999: 43.1; con corrección de lectura de Ferrer 2008a). El nuevo testimonio de Ca n'Oliver acabaría de confirmar que el ibérico puede prescindir en ocasiones de las marcas sufijales, un hecho para el que no es todavía posible dar una explicación concluyente, pero que muy probablemente obedezca a algún tipo de fenómeno sintáctico o gramatical.

ekia: palabra que ha de ser relacionada con el conocido egiar, aunque con dos particularidades: el uso del silabograma oclusivo sordo ( $k i$ ) y no sonoro (gi), como sería más habitual, y la elisión de la vibrante final. Ambas anomalías podrían ser atribuidas a un simple lapsus del escriba.
En efecto las vacilaciones entre silabogramas complejos y simples -una diferenciación que irá paulatinamente cayendo en desuso hasta llegar a desaparecer a principios del s. II a. C. (Ferrer 2005: 971-973)- son relativamente frecuentes. Sin embargo, no es tampoco imposible que pueda tratarse de una variación de tipo fonético o incluso gramatical. Concretamente, la desaparición de la vibrante ante ḿi podría ser también explicada como un fenómeno de caída de vibrante ante nasal en sílaba cerrada, del que tenemos otros ejemplos, como bilostibaś:aḿi (B.1.274), que remite sin duda a bilostibaś-a[r]-ḿi, una típica inscripción de propiedad. Otra explicación alternativa sería que tanto la ausencia de -de en el nombre precedente (vid. supra) como la variación egiar / ekia fueran el resultado de un mismo fenómeno de cohesión gramatical o sintáctica, imposible, sin embargo, de precisar en el estado actual de conocimiento del ibérico.

En cuanto a la interpretación de esta forma, es comúnmente aceptado, como decíamos, que egiar es la palabra característica de las inscripciones de autoría, a 


\begin{tabular}{|c|c|c|c|c|c|c|c|c|}
\hline & NP & $\begin{array}{l}\text { Sufijo de } \\
\text { genitivo }\end{array}$ & $\begin{array}{l}\text { Nombre } \\
\text { común }\end{array}$ & Det. & & $\begin{array}{l}\text { Nombre } \\
\text { común / } \\
\text { Pronombre }\end{array}$ & & Ref. \\
\hline 1. & taŕbanikoŕ & & & & - ́́i & & & F.2.2 \\
\hline 2. & tatứr & -en & & & - & & & B.4.9 \\
\hline 3. & bantor & -en & & & $-\mathbf{m i ́}$ & baikar & & Panosa, $1999,18 \mathrm{~d} .2$ \\
\hline 4. & tikirsbalauŕ & -ar & & & -ḿi & ban & ḿi & C. 4.2 \\
\hline 5. & iltiŕbikis & -en & seltar & & - $\mathbf{m i}$ & & & F.5.1 \\
\hline 6. & & & kaśtaum & ban & $-\dot{m i}$ & & & Burch et al., 2001 \\
\hline
\end{tabular}

Fig. 6: -ḿi tras formas nominales.

\begin{tabular}{|c|c|c|c|c|c|c|c|c|}
\hline & Nombre común & Det. & & NP & Sufijo de agente & ¿Verbos? & & Ref. \\
\hline 7. & seltar & -ban & - $\mathbf{m i}$ & basibalkar & & ḿbar & ḿi & F.14.1,5/6 \\
\hline 8. & & & & nérsetikan & -t(e) & ekiar & ḿi & F.15.1 \\
\hline 9. & & & & & & iunsir & ḿi & F.9.7 \\
\hline
\end{tabular}

Fig. 7: -ḿi tras posibles formas verbales.

menudo, aunque no de forma exclusiva ${ }^{6}$, en el contexto de artesanos que firman sus producciones. Esta interpretación congenia bien, una vez más, con las características de esta nueva inscripción, realizada con el barro aún tierno y relacionable, por lo tanto, con el proceso de elaboración del recipiente. Más controvertido es, sin embargo, el análisis gramatical concreto de la palabra: algunos autores consideran que ha de tratarse de un sustantivo, quizás de contenido verbal, con el sentido de 'obra, producción"7, aunque la opinión mayoritaria es que se trate de una forma verbal de sentido similar al latín fecit o, con valor causativo, faciendum curavit (Correa 1994: 282; Untermann 1994: 127; Rodríguez Ramos 2004: 274-276; Rodríguez Ramos 2005: 43-44; Ferrer 2006: 154-155; Moncunill 2007: 156-58; Velaza 2011: 300), interpretación a la que nos sumamos. Como veremos a continuación, a pesar de que la asociación entre $\boldsymbol{e} \boldsymbol{k i \boldsymbol { a }}$ y $\boldsymbol{m} \boldsymbol{i}$ podría, en efecto, recordar algunas estructuras de tipo nominal, del tipo seltarmí, esta partícula se encuentra también tras formas que admiten un análisis como verbales (fig. 7).

ḿi: se trata de una palabra muy bien documentada en ibérico. Para su interpretación se han planteado distintas hipótesis, como por ejemplo que se trate del verbo copulativo, en $1^{\mathrm{a}}$ o $3^{\mathrm{a}}$ persona, de un pronombre demostrativo o de un pronombre personal en $1^{\text {a }}$ persona $(M L H$ III $\S$ 533; Ferrer 2006: 148-150; Moncunill 2007: 241-242; de Hoz 2011: 260-66). Aunque la cuestión no puede considerarse todavía cerrada, la última opción es tal vez la que permite explicar de forma más coherente todas las distintas apariciones de la palabra, que sintetizamos en las tablas de las figs. 6 y 7.
La primera de ellas recoge los distintos contextos de aparición de -ḿi en relación con un sintagma nominal. Se observa que la palabra puede aparecer:

1. en aposición directa a un NP;

2. como núcleo nominal rigiendo un NP en genitivo;

3 y 4 . como núcleo nominal rigiendo un genitivo y segui-

do de una aposición;

5. en aposición a un núcleo nominal que rige un genitivo; 6. en aposición a un sintagma nominal.

En todos estos casos -ḿi sería compatible tanto con un verbo copulativo (las aposiciones serían entonces atributos) como con un pronombre, ya sea personal o demostrativo. Sin embargo, el primer caso, en el que -mí se pospone directamente al nombre personal, sería más difícilmente explicable como un demostrativo que como un pronombre personal. Por otra parte, teniendo en cuenta su combinación con -ban ( $c f$. no 6), morfo que nunca aparece tras nombres personales, y que es muy frecuente, en cambio, tras palabras del léxico común, la interpretación como pronombre personal de primera persona se perfila como más adecuada, por una mera cuestión de distribución de las funciones. La segunda tabla, muestran ahora los contextos de aparición de -mí tras posibles formas verbales ${ }^{8}$, probablemente ejerciendo la función gramatical de objeto. En este contexto, una interpretación de -ḿi como verbo parece más difícilmente aceptable. Podría tratarse, a lo sumo, de una forma auxiliar, pero sería difícil explicar entonces por qué las mismas formas verbales que la preceden pueden aparecer igualmente de forma independiente, sin producirse cambios importantes ni en la morfología 


\begin{tabular}{|c|c|c|c|c|c|}
\hline NP & Sufijo & ¿Verbo o sustantivo verbal? & ¿Pron? & Cronología & Ref. \\
\hline biloIs]arker & $-d e$ & egiar & & s. III a. C? & Álvarez y Conde 1990, 191-196 \\
\hline jer & & tagiar & & s. III-II a. C. & Francès et al., 2008,223-224 \\
\hline adintar & & ekia & mi & s. III-II a. C. & Inédita \\
\hline
\end{tabular}

Fig. 8: Variantes de la fórmula de autoría documentadas en Ca n’Oliver.

de la palabra ni en la estructura general de la frase. Como argumento adicional cabe aducir, como observa Ferrer (2006: 150), que la coincidencia de la misma forma flexiva de -mí en función de caso "absolutivo" (fig. 6) y de objeto (fig. 7) sería coherente con una estructura de lengua ergativa, como se supone que es el ibérico (Orduña 2008: 275-302; Luján 2002: 289-301). En este caso sería esperable que el sujeto no agente y el objeto de un verbo transitivo presentaran la misa forma.

En consecuencia, una de las interpretaciones que toma más consistencia es que -míi se corresponda con un pronombre personal de $1^{\text {a }}$ persona, en esta ocasión en función de complemento directo de egiar y haciendo alusión probablemente al objeto. Si esta interpretación fuera correcta, nos encontraríamos ante un titulus loquens, cuyo sentido aproximativo podría ser "Adintar me hizo". Como ya hemos dicho, una estructura muy similar se conocía ya en ibérico, en una inscripción realizada sobre un punzón de hueso de Peña de las Majadas (Castellón), con el texto nérsetikan-te-(e)kiar-mí[ (F.15.1).

Finalmente, es interesante resaltar que en el propio yacimiento de Ca n'Oliver se conocen otras dos inscripciones de autoría, con los textos bilo[s]aŕkerdegiar (Álvarez y Conde 1990: 191-196) y ]er tagiar iasbi[ (Francès et al. 2008: 223-224); las estructuras que se definen son parecidas pero presentan diferencias en cada caso: la primera es esquematizable como NP-de+egiar, la segunda como NP-ta+(e)giar o quizá NP-t(e)+agiar. Esta última es especialmente interesante puesto que hasta su aparición esta variante se conocía únicamente en sellos doliares del sur de Francia, hecho por el que se había planteado la posibilidad de que se tratara de una variante local del mejor conocido NP-de-egiar (Ferrer 2008b: 81-93; Moncunill 2007: 291). No obstante, el hecho de que en un mismo entorno convivan las expresiones formadas sobre egiar con aquellas formadas sobre tagiar dificulta que puedan tratarse de variantes diatópicas y hace preferible, en cambio, recorrer a una explicación de tipo gramatical ${ }^{10}$, para la que, sin embargo, todavía no somos capaces de dar una explicación concreta $^{11}$.

\section{CONCLUSIÓN}

Esta nueva inscripción sobre un fragmento de cálato datable a finales del s. III o principios del II a.C. se suma a un importante conjunto de inscripciones ibéricas que encajan en una interpretación como textos de autoría. En el propio yacimiento de Ca n'Oliver, centro con una destacable actividad artesanal y comercial, se conocen, con la que aquí presentamos, un total de tres inscripciones de este tipo, aunque la fórmula documentada no es exactamente la misma en ninguno de los tres casos.

El ejemplar aquí estudiado presenta algunas particularidades gráficas y gramaticales que le confieren un especial interés: 1 . el nombre personal no va seguido, como es habitual, del sufijo -de, comúnmente interpretado como marca de agente; 2 . la forma egiar ha sido escrita de forma defectiva, sin la vibrante final, y con la variante compleja del silabograma $\boldsymbol{k i}$ (ekia); 3 . se documenta aquí por segunda vez la forma -ḿi directamente unida a ekiar. En cuanto al contenido concreto de la fórmula, los paralelos existentes permitirían una posible interpretación del texto como "Adintar me hizo", a pesar de que, teniendo en cuenta el estadio actual de desciframiento del ibérico, toda propuesta de traducción debe ser tomada con la debida cautela

\section{NOTAS}

1. Este trabajo ha recibido fondos de la Unión Europea a través del programa de investigación e innovación Horizon 2020 Marie Curie Actions (IF Marie Sklodowska-Curie grant agreement $\mathrm{N}^{\circ} 655938$ ) y ha sido realizado en el marco del Proyecto de Investigación FFI2015-63981-C3-1-P (Hesperia: lenguas, epigrafía y onomástica paleohispánica) del Ministerio de Economía y Competitividad. Agradecemos a los evaluadores anónimos de este trabajo sus comentarios, que han permitido mejorar el resultado final del texto.

2. Las convenciones tipográficas empleadas para transcribir el ibérico son las siguientes: negrita redonda para los textos escritos en signario ibérico no dual (neitinke), negrita cursiva para los textos en signario dual (baidesbi) y cursiva para los textos grecoibéricos (naltinge). La citación de las inscripciones paleohispánicas se hace según el corpus de Untermann (Monumenta Linguarum Hispanicarum, Wiesbaden 1975-1997 = MLH); para los textos publicados con posterioridad a la aparición de esta obra se proporciona la referencia de la editio princeps. 
3. Para un análisis crítico de la equiparación del ibérico ekiar con el verbo vasco, vid. de Hoz, 2011: 312-113, quien considera inviable una interpretación vasco-iberista de esta forma.

4. Para un estudio de conjunto los grafitos griegos e ibéricos descubiertos hasta la fecha en Ca n'Oliver, vid. Francès et al. 2008: 217-242.

5. Esta misma estructura podría encontrarse también en Jgiegon egiar (F.13.29), aunque la lectura es dudosa.

6. Recuérdese, en este sentido, la aparición de esta fórmula también en la leyenda monetal aŕsbigisdeegiar en una dracma de Arse (A.33.2), donde parece mencionarse el magistrado encargado de la emisión.

7. Así, por ejemplo, de Hoz 2001: 353-357; Orduña 2009 501-503; Quintanilla 2005: 513 (lo considera un sustantivo que podría actuar como verbo). Para una interpretación como forma nominal del verbo, vid. Luján 2010: 239; Orduña 2010: 332.

8. Para la identificación del verbo en ibérico vid. Velaza 2011: 295-304.

9. A lo largo de los últimos años han aparecido en Ca n'Oliver varias piezas cerámicas con una decoración muy similar a la de esta pieza. Todas estas piezas son agrupables en la fase $3 \mathrm{~B}$ del yacimiento, correspondiente a finales del s. III y principios del s. II a.C. Los demás grafitos del poblado con procedencia estratigráfica conocida pertenecen también a esta fase.

10. Si la lectura latubaŕentagiar (B.1.364) se confirmara, sería posible postular una típica estructura de posesión del tipo NP-en+sustantivo. Una posible interpretación sería entonces considerar que tagiar se correspondiera precisamente con un sustantivo verbal derivado de egiar.

11. Con todo, el hecho de que los tres grafitos hayan aparecido en Ca n'Oliver no hace estrictamente imposible que hayan sido producidos, y quizás también inscritos, en lugares distintos. La presencia de hornos y de actividad artesanal en el mismo yacimiento parece indicar que se trate, en efecto, de producciones locales, pero solo un estudio pormenorizado de las características de las pastas cerámicas permitiría afirmarlo con total seguridad.

\section{BIBLIOGRAFÍA}

ÁLVAREZ, R.; CONDE, M. J. (1990): Aportació a l'estudi de les ceràmiques grises ibèriques decorades: dos exemples concrets, Actes del VIII Col-loqui Internacional d'Arqueologia de Puigcerdà, Puigcerdà, 191-196.

BARRIAL, O.; FRANCÈS, J. (1985): Les escultures ibèriques zoomorfes del Turó de Ca n'Olivé (Cerdanyola del Vallès, Vallès Occidental), Empúries 47, 254-263.

BELTRÁN, P. (1942): Sobre un interesante vaso escrito de San Miguel de Liria, Serie Trabajos Varios. S.I.P. 8, València.

BURCH, J.; NOLLA, J. M.; PALAHÍ, Ll.; SAGRERA, J.; SUREDA, M.; VIVÓ, D. (2001): Excavacions arqueològiques a la muntanya de Sant Julià de Ramis. 1. El sector de l'antiga església parroquial, Sant Julià de Ramis.

CORREA, J. A. (1994): La lengua ibérica, Revista española de lingüística 24 (2) 263-287.
DE HOZ, J. (2001): Hacia una tipología del ibérico, Religión, lengua y cultura prerromanas de Hispania. Actas del VIII Coloquio sobre lenguas y culturas paleohispánicas (F. Villar, P. Fernández, eds.), Salamanca, 335-362.

DE HOZ, J. (2011): Historia Lingüística de la Península Ibérica en la Antigüedad. II: El mundo ibérico prerromano y la indoeuropeización, Manuales y Anejos de Emerita 51, Madrid.

FERRER, J. (2005): Novetats sobre el sistema dual de diferenciació gràfica de les oclusives sordes i sonores, Acta Palaeohispanica IX: Actes del IX Col-loqui Internacional de Llengües $i$ Cultures Paleohispàniques (Barcelona 20-24 d'octubre de 2004) [= PalHisp 5], (F. Beltrán, C. Jordán, J. Velaza, eds.), Zaragoza, 957-982.

FERRER, J. (2006): Nova lectura de la inscripció ibèrica de la Joncosa (Jorba, Barcelona), Veleia 23, 129-170.

FERRER, J. (2008a): Ibèric tagiar. Terrissaires que signen les seves produccions: biuŕrko, ibeitigeŕ, biuŕbedi i companyia, $S E$ Barc VI, 81-93.

FERRER, J. (2008b): Ibèric kaśtaun: un element característic del lèxic sobre torteres, Cypsela 17, 253-271.

FERRER, J.; ESCRIVÀ, V. (2013): Quatre noves inscripcions ibèriques pintades procedents de Llíria, Palaeohispanica 13 461-482.

FRANCES, J.; VELAZA, J.; MONCUNILL, N. (2008): Los esgrafiados sobre cerámica de Ca n'Oliver (Cerdanyola del Vallès), Palaeohispanica 8, 217-242.

FRANCÈS, J.; GUÀRDIA, M. (2011): El poblat ibèric del Turó de Ca n'Oliver (segles VI - I aC). Balanç de 25 anys de recerca i difusió, Tribuna d'Arqueologia, 267-291.

GÓMEZ MORENO, M. (1945): Digresiones ibéricas: escritura, lengua, Boletín de la Real Academia Española XXIV, 275-288.

HESPERIA = Banco de Datos Hesperia de Lenguas Paleohispánicas, http://hesperia.ucm.es/

LUJÁN, E. R. (2010): Las inscripciones musivas ibéricas del valle medio del Ebro: una hipótesis lingüística, Palaeohispanica 10, 291-298.

$M L H=$ Untermann, J. (1975-1997): Monumenta Linguarum Hispanicarum, Wiesbaden.

MARGENAT, F. (2015): Les esteles ibèriques amb representacions de llances. El cas de Rubí, Butlletí del Grup de Col-laboradors del Museu de Rubí. 65, 2-21.

MONCUNILL, N. (2007): Lèxic d'inscripcions ibèriques, Universitat de Barcelona.

MONCUNILL, N. (2017): Nombres comunes en ibérico y su inserción dentro de la frase, Palaeohispanica XVII, e.p.

ORDUÑA, E. (2008): Ergatividad en ibérico, Emerita 76, 275-302.

ORDUÑA, E. (2009): De nuevo sobre el sufijo ibérico -te, Acta Palaeohispanica X. Actas do X Colóquio internacional sobre Linguas e Culturas Paleo-hispânicas (Lisboa, 26-28 de Fevereiro de 2009) [= PalHisp 9] (F. Beltrán, J. D’Encarnação, A. Guerra, C. Jordán, eds.), Zaragoza, 501-514. 
ORDUÑA, E. (2010): En torno al lexema ibérico eki- y sus variantes, Palaeohispanica 10,319-334.

PANOSA, M. I. (1999): La escritura ibérica en Cataluña y su contexto socioeconómico (siglos V-I a.C.), Vitoria.

QUINTANILLA, A. (2005): Palabras de contenido verbal en ibérico, Acta Palaeohispanica IX: Actes del IX Col-loqui Internacional de Llengües i Cultures Paleohispàniques (Barcelona 20-24 d'octubre de 2004) [= PalHisp 5], (F. Beltrán, C. Jordán, J. Velaza, eds.), Zaragoza, 507-522.

RODRÍGUEZ RAMOS, J. (2004): Análisis de epigrafía íbera, Vitoria-Gasteiz.
RODRÍGUEZ RAMOS, J. (2005): Introducció a l'estudi de les inscripcions ibèriques, Barcelona.

UNTERMANN, J. (1994): Comentario a la inscripción musiva de Andelos, Trabajos de Arqueología de Navarra 11, 127-129.

VELAZA, J. (2002): Ibérico -te, Palaeohispanica 2, 271-275.

VELAZA, J. (2011): Cuestiones de morfología verbal en ibérico, A Greek man in the Iberian street. Papers on linguistics and epigraphy in honour of Javier de Hoz (J. L. García Alonso, E. R. Luján, eds.), Innsbruck, 295-304. 